\title{
Oral hygiene in elderly people in hospitals and nursing homes
}

\author{
Abstracted from \\ Sjögren P, Nilsson E, Forsell M, Johansson O, Hoogstraate J. \\ A systematic review of the preventive effect of oral hygiene on pneumonia and respiratory tract infection in \\ elderly people in hospitals and nursing homes: effect estimates and methodological quality of randomised controlled trials. \\ I Am Geriatr Soc 2008; 56: 2124-2130
}

Address for correspondence: Dr Petteri Sjögren, Oral Care AB, Drottninggatan 13,

SE-411 15 Göteborg, Sweden. E-mail: petteri.sjogren@oralcare.se

\section{Question: Does improved oral hygiene lead to reductions in cases of pneumonia and respiratory tract infections in elderly people in hospitals and nursing homes?}

Data Sources Searches were made using Medline, the Cochrane Library, the National Health Service Economic Evaluation database and, by hand, of relevant articles' reference lists.

Study selection The search was limited to studies conducted in humans only. The predetermined inclusion criteria were: clinical studies and randomised controlled trials (RCT) that linked oral hygiene with healthcare-associated pneumonia or respiratory tract infection in elderly people. Publications in Dutch, English, German and any of the Nordic languages (Danish, Finnish, Icelandic, Norwegian, Swedish) were included. Articles about authority opinions and reports of expert committees were excluded, as were studies on subjects who required mechanical ventilation or tube feeding.

Data extraction and synthesis Data extraction from RCT was focused on the outcomes. Assessments were made of the quality and validity of the studies, using statistical methods to test these. To ensure the consistency of the assessments throughout the study, two authors (EN, PS) performed the data extraction independently, and any disagreements were resolved in consensus meetings. Pooling data from individual studies (meta-analysis) was not deemed appropriate because of heterogeneous study designs, quality of reporting methodological aspects and trial conduct. To provide an overview of additional clinical studies in this research area, the non-RCT studies that were identified were scrutinised for the authors' main conclusion(s).

Results Fifteen publications, of which five were RCT, fulfilled the inclusion criteria and were considered throughout all the assessments. All of the RCT revealed positive preventive effects of oral care on pneumonia or respiratory tract infection in nursing home residents, with numbers needed to treat ranging from 8.6-15.3.

Conclusions Available results from RCT provide evidence that mechanical oral hygiene decreases mortality risk from pneumonia and seems to have a clinically relevant preventive effect on nonfatal pneumonia in independent elderly individuals. The data show that providing mechanical oral hygiene may prevent approximately 1 in 10 cases of death from pneumonia in dependent elderly people and indicate a largely similar effect for prevention of pneumonia.

\section{Commentary}

The literature tells us that institutionalised dependent seniors living in a hospital setting or in a nursing home display very poor oral health. Preceding generations reached an advanced age with few or no teeth left in their mouths. With the progress made in oral hygiene, however, and the increased importance given to oral health and the accessibility of dental services, seniors are keeping their natural teeth longer and longer. The literature review also demonstrates the strong prevalence of pneumonia and respiratory tract infections in this population. The question posed by Sjögren et al. is thus extremely pertinent for learning about the preventive effect of oral hygiene on pneumonia and respiratory tract infections in institutionalised seniors.

For this purpose, the authors identified 15 articles that met their inclusion criteria. From these, only one systematic review and five RCT were selected and used. Two of these RCT covered the same study. The authors also used non-RCT studies they had identified in order to draw their conclusions.

The authors evaluated the methodological quality of their selected studies, examining the following: heterogeneity, method of random allocation, double-blinding, withdrawals and dropouts, quality score, power calculation, definition of the studied endpoint and the trial design. The quality of the studies varied, and most were not of high quality.

Sjögren and colleagues concluded that mechanical oral hygiene has a preventive effect on mortality caused by pneumonia. Some reservations must be expressed about this conclusion: it is based on two studies only, and in particular, their systematic review of the existing literature shows us the methodological limitations of the studies carried out thus far.

A critical reading of the systematic review is always from the individual reader's point of view, whether they be practitioner, researcher or even public-health decision-maker. Depending on the point of view, such a systematic review may appear somewhat questionable or somewhat informative. For the researcher, it is obvious that other clinical studies with strict protocols are vital. In the meantime, the results of this review indicate to the clinician and the decisionmaker that it would be desirable for practice guidelines specifying healthcare required for institutionalised seniors to include specific oral hygiene interventions.

Sophie Arpin Direction de la Santé Publique de la Montérégie, Longueuil, Province of Quebec, Canada

Evidence-Based Dentistry (2009) 10, 46. doi:10.1038/sj.ebd.6400649 